\title{
Entanglement Capability of Two-qubit Operations
}

\author{
B. Kraus, W. Dür, G. Vidal, J. I. Cirac, M. Lewenstein ${ }^{\mathrm{a}}$, N. Linden ${ }^{\mathrm{b}}$, and S. Popescu ${ }^{\mathrm{c}, \mathrm{d}}$ \\ Institut für Theoretische Physik, Universität Innsbruck,A-6020 Innsbruck, Austria \\ ${ }^{a}$ Institut für Theoretische Physik, Universität Hannover, Hannover, Germany \\ ${ }^{b}$ Department of Mathematics, University of Bristol, University Walk, Bristol BS8 1TW, UK \\ ${ }^{c}$ H. H. Wills Physics Laboratory, University of Bristol, Tyndall Avenue, Bristol BS8 1TL, UK \\ ${ }^{\mathrm{d}}$ BRIMS, Hewlett-Packard Laboratories, Stoke Gifford, Bristol BS12 6QZ, UK
}

Reprint requests to B. K.; Fax: +43 512 507291939; E-mail: Barbara.Kraus@uibk.ac.at

Z. Naturforsch. 56 a, 91-99 (2001); received February 6, 2001

Presented at the 3rd Workshop on Mysteries, Puzzles and Paradoxes in Quantum Mechanics, Gargnano, Italy, September 17 - 23, 2000.

\begin{abstract}
We study the optimal way of creating bi-partite entanglement using a general two-qubit interaction. On the one hand, we analyze the entanglement capability of an arbitrary non-local Hamiltonian acting on two qubits. We explicitly calculate the state which maximizes the entanglement produced per time step $\delta t$ during the non-local evolution. On the other hand, we determine the maximal amount of entanglement which can be produced by an arbitrary two-qubit gate. We also give the separable state which leads to the output state containing this amount of entanglement. Furthermore, we consider the situation where auxiliary systems are present. Finally, we determine the non-unitary processes which are able to create entanglement from an initially separable state of two systems. - Pacs: 03.67.-a, 03.65.Bz, 03.65.Ca, 03.67.Hk
\end{abstract}

Key words: Entanglement; Quantum Information; Quantum Gates.

\section{Introduction}

Entanglement is one of the most fascinating features of quantum mechanics [1, 2], and at the same time, a highly preciated resource in quantum information science. In recent years, considerable effort has been devote to create entanglement in the laboratory [3]. In some of these experiments entanglement is produced by starting out from a product state of two systems (typically qubits) and using some physical process that gives rise to an interaction between them. Thus, one of the relevant problems in this context is to find ways of generating "as much entanglement as possible" for a given experimental set-up, i. e. a non-local interaction.

The first steps towards answering this problem have been given in [4 - 7]. In [5] we considered an arbitrary non-local Hamiltonian $H$ acting on two qubits. The time evolution induced by $H$ changes the (possibly entangled) state of the two qubits and in particular its entanglement. For any given amount of initial entanglement $E$, we first determined the state $\left|\Psi_{E}\right\rangle$ for which more entanglement $\delta E$ is produced after a time step $\delta t$, and then the amount of initial entanglement $E_{0}$ which leads to the maximal entanglement production. Remarkably, a unique parameter, the entanglement capability of the two-qubit Hamiltonian $H$, determines the optimal $\delta E$ for any initial amount of entanglement $E$. This quantity, that we computed for an arbitrary $H$, completely characterizes how efficient $H$ is at producing entanglement. It also turned out that, for finite-time evolutions, the optimal entanglement production requires that we apply local unitary operators after each time step $\delta t$. In some situations, however, one cannot apply fast local operations during the process, but rather a fixed quantum gate is given. This problem was analyzed in [6]. There we determined the separable input state which maximizes the entanglement of the state obtained after applying an arbitrary two-qubit gate. These results allow us to characterize arbitrary two qubit interactions in terms of the entanglement which they can produce. For example, we determined which are the two-qubit gates that can create maximally entangled 
states. While most of our results are concerned with two qubits, we also showed that if we allow the latter to be initially (locally) entangled with some ancillas, one can obtain more entanglement, at least for certain measures of entanglement. A natural question in this context is: which physical actions (not necessary unitary) are able to create entanglement? In [7] we answered this question using an isomorphism between physical actions and density operators. This powerful one-to-one correspondence allows us to translate all the known properties of density operators to the ones of physical processes.

This paper can be viewed as a unified summary of [5], [6] and [7]. We show how much entanglement can be produced by an arbitrary non-local two-qubit interaction and determine the input states which lead to this amount of entanglement. We also define the entanglement capability of an arbitrary Hamiltonian acting on two qubits. In the case where we allow for auxiliary systems we discuss the maximal entanglement production for certain measures of entanglement. Finally, we show which non-unitary processes are able to create entanglement.

This paper is divided into five sections. In Sect. II we recall some definitions and introduce some notation. In Sect. III we study how much entanglement can be created by a two-qubit unitary operation. This section is divided into two parts. In the first one we analyze the maximum amount of entanglement which can be created by an arbitrary non-local Hamiltonian if we are allowed to apply fast local unitary operations during the evolution. In the second part we show how much entanglement can be produced by an arbitrary two-qubit gate. In both cases we will determine the initial state which maximizes the entanglement production. In Sect. IV we consider the situation where Alice and Bob have more than one qubit each, which they are allowed to entangle initially locally. We will see there with some examples that the question of the best input state is not well-defined since the answer, in this case, depends on the measure of entanglement. Once we have specified the measure of entanglement we will show that for some two-qubit operations, auxiliary systems will help to create more entanglement. In Sect. V we analyze general (not necessary unitary) physical processes. We develop an isomorphism between physical actions and density operators acting on two systems, which allows us to decide when a physical process is able to create entanglement out of an initially separable state.

\section{Definitions and Measures of Entanglement}

This section is divided into two parts. In the first one we recall some definitions and thereby introduce our notation. In the second part we recall some measures of entanglement.

\section{II.A Definitions}

Throughout this paper we consider two systems $A$ and $B$ at different locations, held by Alice and Bob, respectively. These systems will be composed of one or two qubits each. Their states are represented by vectors in the Hilbert space $\mathcal{H}_{A, B}$ respectively.

We will use the following maximally entangled state of the two systems, of $d$ dimensions each,

$$
|\Phi\rangle_{A B}=\frac{1}{\sqrt{d}} \sum_{i=1}^{d}|i\rangle_{A} \otimes|i\rangle_{B},
$$

where $\{|i\rangle\}_{i=1}^{d}$ is an orthonormal basis in $\mathcal{H}_{A}$ and $\mathcal{H}_{B}$.

For two qubits, a basis of $\mathcal{H}_{A} \otimes \mathcal{H}_{B}$ which contains only orthogonal maximally entangled states is the Bell basis. It is defined as

$$
\left|\Phi^{ \pm}\right\rangle=\frac{1}{\sqrt{2}}(|00\rangle \pm|11\rangle),\left|\Psi^{ \pm}\right\rangle=\frac{1}{\sqrt{2}}(|01\rangle \pm|10\rangle) \text {. }
$$

We also make use of the so-called magic basis [8], which is defined in the same way as the Bell-basis, except for some global phases. We will denote the elements of this basis by

$$
\begin{aligned}
& \left|\Phi_{1}\right\rangle=\left|\Phi^{+}\right\rangle,\left|\Phi_{2}\right\rangle=-i\left|\Phi^{-}\right\rangle, \\
& \left|\Phi_{3}\right\rangle=\left|\Psi^{-}\right\rangle,\left|\Phi_{4}\right\rangle=-i\left|\Psi^{+}\right\rangle .
\end{aligned}
$$

Throughout this paper we use capital Greek letters for joint states of systems $A$ and $B$ and small letters for states describing either system $A$ or system $B$. We denote by $\left|\Psi^{\perp}\right\rangle$ a state which is orthogonal to $|\Psi\rangle$, whereas $\left|\Psi^{*}\right\rangle$ denotes the complex conjugate of $|\Psi\rangle$ in the computational basis, $\{|0\rangle,|1\rangle\}$. We will denote the Pauli operators by $\sigma_{1}, \sigma_{2}, \sigma_{3}$ and $\sigma$ denotes the vector whose elements are the Pauli operators. Furthermore $\sigma^{\mathrm{T}}$ denotes the transpose of $\sigma$. If it is not clear which system an operator is acting on, we specify it with either a sub- or superscript.

\section{II.B Measures of Entanglement}

For bipartite systems several measures of entanglement have been proposed, each one inducing a differ- 
ent ordering in the set of entangled states. Nonetheless, they all agree that (1) are the maximally entangled states, and they all vanish for product (i. e. separable, or non-entangled) states. Let us now review some of these measures of entanglement for pure states of two systems. We start out with the so-called concurrence [9], $C$, which is a measure of entanglement for states of two qubits. It is defined as

$$
C(|\Psi\rangle)=\left|\left\langle\Psi\left|\sigma_{2} \otimes \sigma_{2}\right| \Psi^{*}\right\rangle\right| .
$$

Writing $|\Psi\rangle$ in the magic basis, i.e $|\Psi\rangle=$ $\sum_{k=1}^{4} \mu_{k}\left|\Phi_{k}\right\rangle$, we obtain

$$
C(|\Psi\rangle)=\left|\sum_{k} \mu_{k}^{2}\right| .
$$

Let us note here that $C(|\Psi\rangle)=1$, that is $|\Psi\rangle$ is maximally entangled, if $\mu_{k}^{2}=e^{i \delta}\left|\mu_{k}\right|^{2} \forall k$. On the other hand $C(|\Psi\rangle)=0$, that is $|\Psi\rangle$ is a product state, if $\sum_{k} \mu_{k}^{2}=0$.

The rest of measures, we will make use of, are better described in terms of the Schmidt coefficients. A pure state $|\Psi\rangle$, describing the state of two systems, $A$ and $B$, each of dimension $d$, always has a Schmidt decomposition in the form

$$
|\Psi\rangle=\sum_{k=1}^{m} c_{k}\left|\phi_{k}\right\rangle_{A}\left|\psi_{k}\right\rangle_{B},
$$

where $m \leq d$ and $\left\langle\phi_{k} \mid \phi_{l}\right\rangle=\left\langle\psi_{k} \mid \psi_{l}\right\rangle=\delta_{k l}$ $\forall k, l=1, \ldots, m$. The real and positive coefficients $c_{k}$, which are the square roots of the eigenvalues of the reduced density operator, $\rho_{A}=\operatorname{tr}_{B}(|\Psi\rangle\langle\Psi|)$ (or $\rho_{B}=\operatorname{tr}_{A}(|\Psi\rangle\langle\Psi|)$ ), are called Schmidt coefficients. We will choose them in decreasing order, i.e. $c_{1} \geq c_{2} \ldots \geq c_{m}>0$.

The Entropy of entanglement is defined as

$$
\begin{aligned}
E_{E}(|\Psi\rangle) \equiv S\left(\rho_{A}\right) & =-\operatorname{tr}\left[\rho_{A} \log _{2}\left(\rho_{A}\right)\right] \\
& =-\sum_{k=1}^{m} c_{k}^{2} \log _{2}\left(c_{k}^{2}\right) .
\end{aligned}
$$

This measure has an interesting operation meaning: given $n$ copies of a state $|\Psi\rangle$, then one can produce, using only local operations and classical communication, $n E_{E}(|\Psi\rangle)$ maximally entangled states of two qubits and vice versa (in the limit $n \rightarrow \infty$ ) [11].

Another useful measure is the Schmidt number [10], which we will denote by $E_{\mathrm{S}}$. It is the number of Schmidt coefficients minus one, i. e. $E_{\mathrm{S}}(|\Psi\rangle)=m-1$.
In the context of manipulating a single copy of a bipartite pure state, the set of entanglement monotones [12]

$$
E_{n}(|\Psi\rangle)=\sum_{k=n}^{m} c_{k}^{2},
$$

for $n=1, \ldots, m$, provides all the information about which transformations are possible using only local operations and classical communication, and we will also consider them.

Finally, we will also use the so-called 2-Entropy (related to the 2-Rènyi entropy) of the reduced density operator [13]. It is defined as

$$
E_{\mathrm{R}}(|\Psi\rangle) \equiv S_{\mathrm{R}}\left(\rho_{A}\right)=1-\operatorname{tr}\left(\rho_{A}^{2}\right)=1-\sum_{k=1}^{m} c_{k}^{4} .
$$

In the following we will call this measure the Rènyi entanglement.

Note that any measure of entanglement must only depend on the Schmidt coefficients, given the fact that it must be invariant under local unitary operations. Thus, in the case of two qubits the situation is fairly simple, because a state describing two qubits can have at most two Schmidt coefficients. Due to the normalization only one of these is independent. This implies that in this case all the measures of entanglement are monotonic functions of each other. Note further that two states of two qubits which are equally entangled can always be transformed into each other using local unitary operators. In higher dimensions, however, this is no longer true. There it might happen that, according to some measure of entanglement, a state is more entangled than some other, whereas for some other measure it is the other way around.

\section{Optimal Way of Entangling Two Qubits}

In this section we show how to entangle two qubits in an optimal way. We consider the situation where Alice and Bob have one qubit each. Note that in order to maximize the entanglement production we only have to consider pure states*. We are going to treat

${ }^{*}$ Note that the maximum entanglement reached for pure states is always larger than the one reached by a mixed state. This can be seen as follows: let us write an arbitrary mixed state, $\rho$ in its eigenbasis, i.e $\rho=\sum_{k} p_{k}\left|\Psi_{k}\right\rangle\left\langle\Psi_{k}\right|$. Using the fact that any measure of entanglement, $E$, is convex [14] we have that $E(\rho) \leq$ $\max _{\left|\Psi_{k}\right\rangle} E\left(\left|\Psi_{k}\right\rangle\right)$. Thus it suffices to consider only pure states. 
two different scenarios. In the first part of this section we will show how to create as much entanglement as possible using a non-local Hamiltonian, $H$ [5]. We will allow Alice and Bob to apply some fast local unitary operations to their qubits during the process. From the analysis it will become clear why these intermediate local operations will increase the entanglement production. In the second part of this section we will determine the maximum amount of entanglement which can be produced by a general two-qubit gate, $U$ [6]. Here we will not allow for operations during the process, but rather deal with the situation where Alice and Bob apply a non-local unitary operator to their qubits. In both cases we will also determine the state which Alice and Bob have to use initially in order to create, given a certain non-local interaction, as much entanglement as possible. In the first case it will turn out that, independently of the Hamiltonian it is better to start with some initially entangled state. In the second case we only consider initially separable states. The method used there can be easily generalized to initially entangled states.

\section{III.A Entanglement Capability of Non-local Hamiltonians}

We will characterize the entanglement of a state at a given time $t,|\Psi(t)\rangle$, by some entanglement measure $E$. In order to quantify the entanglement production, we define the entanglement rate $\Gamma$ at a particular time $t$ of the interaction as

$$
\Gamma(t) \equiv \frac{\mathrm{d} E(t)}{\mathrm{d} t} .
$$

The goal is then to find the conditions which must be satisfied in order to obtain a maximal entanglement rate. In particular, we will be interested in determining the following:

(i) For any initial entanglement $E$ of the two-qubit system, what is the state $|\Psi\rangle$, say $\left|\Psi_{E}\right\rangle$, for which the interaction produces the maximal rate $\Gamma_{E}$.

(ii) The maximal achievable entanglement rate $\Gamma_{\max }$,

$$
\Gamma_{\max } \equiv \max _{E} \Gamma_{E}
$$

and the state $\left|\Psi_{\max }\right\rangle$ for which $\Gamma=\Gamma_{\max }$.

In order to explain why those quantities are interesting, let us write the state of the qubits, $|\Psi(t)\rangle$, in its Schmidt decomposition (6)

$$
|\Psi\rangle=\sqrt{P}|\varphi, \chi\rangle+e^{i \alpha} \sqrt{1-P}\left|\varphi^{\perp}, \chi^{\perp}\right\rangle,
$$

where for the sake of short-hand notation we have omitted the time dependence of all these quantities. We take $P \leq 1 / 2$. Note that, as mentioned in Sect. II.B, any measure of entanglement, $E$, must only depend on the Schmidt coefficient $P$, so we write $E(P)$ for some given measure of entanglement. Given now some value $E$, let $\left|\Psi_{E}\right\rangle$ be the state with entanglement $E$ and in which $|\varphi\rangle,|\chi\rangle$ and $\alpha$ are chosen such that $\Gamma_{E}$ is maximized. As explained in Sect. II.B, it is always possible to transform a state of two qubits with entanglement $E$ into $\left|\Psi_{E}\right\rangle$ by local unitary operators. Let the system now evolve under $H$. Then the state after a very small time step $\delta t$ will change its entanglement to $E+\delta E$ and the states $|\varphi\rangle$ and $|\chi\rangle$ will change too. We apply now fast local unitary operators to transform this new state into $\left|\Psi_{E+\delta E}\right\rangle$. Proceeding in the same way after every time step, and taking the continuous time limit $\delta t \rightarrow 0$, we obtain that the state of the qubits at time $t$ is always the optimal one, $\left|\Psi_{E(t)}\right\rangle$.

Knowledge of $\Gamma_{E}$ also permits us to determine the maximum amount of entanglement $E_{\max } \equiv$ $\int_{t_{0}}^{t} \mathrm{~d} t \Gamma_{E(t)}$ produced after a given finite time $t-t_{0}$. We just have to express $\Gamma_{E}$ as an explicit function of $E$, substitute it in (10) and solve that differential equation to determine $E_{\max }(t)$. Note that the optimal procedure described above will precisely reach the entanglement $E_{\max }(t)$.

The state $\left|\Psi_{\max }\right\rangle$ is important since it gives rise to the maximal increase of entanglement, and therefore corresponds to the best operational point. After reaching the state $\left|\Psi_{\max }\right\rangle$ with the procedure described in the previous paragraph, the entanglement would be produced in a very efficient way if one could transfer the entanglement that is gained after each time step $\delta t$ to other qubits (using entanglement dilution [11] or some other means). In particular, the entanglement of the system would increase proportionally to the time, $\Gamma_{\max }$ being the proportionality constant.

In the following, we will show how to determine $\left|\Psi_{E}\right\rangle, \Gamma_{E},\left|\Psi_{\max }\right\rangle$, and $\Gamma_{\max }$ for an arbitrary Hamiltonian $H$. Given the fact that the entanglement, $E$, of the state (12) must only depend on the Schmidt coefficient $P$, we find that

$$
\Gamma(t)=\frac{\mathrm{d} E}{\mathrm{~d} P} \frac{\mathrm{d} P}{\mathrm{~d} t} .
$$


In (13), given a particular entanglement measure $E(P)$, we just have to determine $\mathrm{d} P / \mathrm{d} t$. In order to do so, we need to find the (infinitesimal) time evolution of the Schmidt coefficients of the state of the qubits. Using standard perturbation theory we find [5]

$$
\frac{\mathrm{d} P}{\mathrm{~d} t}=2 \sqrt{P(1-P)} \times \operatorname{Im}\left[e^{i \alpha}\left\langle\varphi, \chi|H| \varphi^{\perp}, \chi^{\perp}\right\rangle\right],
$$

where we have omitted the time-dependence. Upon substitution in (13) we obtain the entanglement rate. Since we are interested in maximizing $\Gamma$, it is clear that we can always choose $\alpha$ such that

$$
\Gamma=f(P)|h(H, \varphi, \chi)|,
$$

where

$$
\begin{aligned}
& f(P)=2 \sqrt{P(1-P)} E^{\prime}(P), \\
& h(H, \varphi, \chi)=\left\langle\varphi, \chi|H| \varphi^{\perp}, \chi^{\perp}\right\rangle .
\end{aligned}
$$

From (15) it is clear that we can maximize $f$ and $h$ independently, since they depend on different parameters. We want to stress that (15) tells us that for any value $E$ of the entanglement, the states $|\varphi\rangle$ and $|\chi\rangle$ for which the maximal entanglement rate $\Gamma_{E}$ is obtained do not depend on $E$, but only on the form of the Hamiltonian $H$. Let us denote by $h_{\max }$ the maximum value of $|h|$; that is,

$$
h_{\max }=\max _{\|\varphi\|,\|\chi\|=1}\left|\left\langle\varphi, \chi|H| \varphi^{\perp}, \chi^{\perp}\right\rangle\right| .
$$

Now, we can easily solve (14) and find $P(t)=$ $\sin ^{2}\left[h_{\max } t+\phi_{0}\right]$, with $P(0)=\sin ^{2}\left(\phi_{0}\right)$. For concreteness sake, in order to maximize now $f$ we choose the entropy of entanglement (7) as a measure of entanglement $E(P)$. As discussed in Sect. II.B, any other two-qubit measure would lead to similar results. With this choice of measure the value of $P_{0}$ that maximizes $f(P)$ has to fulfill

$$
\ln \frac{1-P_{0}}{P_{0}}=\frac{2}{1-2 P_{0}},
$$

i.e. $P_{0} \simeq 0.0832$, which gives $E\left(P_{0}\right) \simeq 0.413$. This shows that, in order to increase the entanglement of a two-qubit system in an optimal way, it is better to start with some initially entangled state rather than a product state. Note that the optimal initial entanglement $E\left(P_{0}\right)$ is independent of the Hamiltonian.
As we have seen above, the evolution of the entanglement is fully characterized by $h_{\max }$. We call this quantity, since it depends only on the interaction Hamiltonian, the entanglement capability of the Hamiltonian, $H$. Let us now show how one can determine this quantity.

Any Hamiltonian can be written (except for a trivial constant) as

$$
H=\left(\boldsymbol{\alpha}^{\mathrm{T}} \boldsymbol{\sigma}_{A}\right) \otimes \mathbb{1}_{B}+\mathbb{1}_{A} \otimes\left(\boldsymbol{\beta}^{\mathrm{T}} \boldsymbol{\sigma}_{B}\right)+\boldsymbol{\sigma}_{A}^{\mathrm{T}} \gamma \boldsymbol{\sigma}_{B} .
$$

Here $\alpha, \beta$, and $\gamma$ are, respectively, two real vectors and a real matrix, and $T$ denotes transposition. Allowing for local unitary operations, in order to determine $h_{\max }$ we only have to consider Hamiltonians of the form

$$
\hat{H}=\sum_{k=1}^{3} \gamma_{k} \sigma_{k}^{A} \otimes \sigma_{k}^{B},
$$

where $\gamma_{1} \geq \gamma_{2} \geq \gamma_{3} \geq 0$ are the (sorted) singular values* of the matrix $\gamma$.

The reason for that is two fold. On the one hand, it is clear that the terms $\left(\boldsymbol{\alpha}^{\mathrm{T}} \boldsymbol{\sigma}_{A}\right) \otimes \mathbb{1}_{B}$ and $\mathbb{1}_{A} \otimes\left(\boldsymbol{\beta}^{\mathrm{T}} \boldsymbol{\sigma}_{B}\right)$ in (19) do not contribute in the expression (17). On the other hand, we can write $\gamma$ in its singular value decomposition, i.e $\gamma=O_{1} \gamma_{d} O_{2}$, where $O_{1}$ and $\mathrm{O}_{2}$ are orthogonal matrices and $\gamma_{d}$ is a positive diagonal matrix. The sorted singular values of $\gamma$ (the diagonal elements of $\gamma_{d}$ ) will be denote by $\gamma_{1}, \gamma_{2}, \gamma_{3}\left(\gamma_{1} \geq \gamma_{2} \geq \gamma_{3} \geq 0\right)$. Now, allowing for local unitary operators, $U_{A}\left(V_{B}\right)$ and $U_{A}^{\dagger}\left(V_{B}^{\dagger}\right)$, before and after the evolution, where $U_{A}^{\dagger} \sigma_{A}^{\mathrm{T}} U_{A}=\sigma_{A}^{\mathrm{T}} O_{1}^{\mathrm{T}}$ $\left(V_{B}^{\dagger} \sigma_{B} V_{B}=O_{2}^{\mathrm{T}} \sigma_{B}\right)$ we find that the Hamiltonian is transformed into the standard form (20). In that case, we obtain that the total (non-local) effect of the evolution for a time $t$ is equivalent to the one obtained with the Hamiltonian $\hat{H}$ for the same time. Using this fact one can show [5] that

$$
h_{\max }=\gamma_{1}+\gamma_{2}
$$

Note that this maximum is reached if $|\chi\rangle \perp|\varphi\rangle$ and $|\varphi\rangle=|0\rangle$ or $|\varphi\rangle=|1\rangle$, i.e. an eigenstate of $\sigma_{3}$.

Summarizing, once we have transformed the Hamiltonian $H$ to the standard form (20) we obtain that for a given value of $E$ (and therefore of $P$ ),

*Given a real matrix $\gamma$ one can always find two orthogonal matrices $O^{1}$ and $O^{2}$ such that $\gamma=O^{1} \mu O^{2}$, where $\mu$ is a diagonal positive matrix. This is called singular value decomposition of $\gamma$. 


$$
\begin{aligned}
& \left|\Psi_{E}\right\rangle=\sqrt{P}|0,1\rangle+i \sqrt{1-P}|1,0\rangle, \\
& \Gamma_{E}=f(P) h_{\max },
\end{aligned}
$$

where $h_{\max }=\gamma_{1}+\gamma_{2}$. The maximum rate $\Gamma_{\max }$ is obtained for $P=P_{0}$, where $P_{0}$ is the value that maximizes $f(P)$. Thus, $\left|\Psi_{\max }\right\rangle$ and $\Gamma_{\max }$ are given by (22) with $P=P_{0}$. For example, for the entropy of entanglement as entanglement measure $P_{0} \simeq 0.0832$, which leads to $f\left(P_{0}\right) \simeq 1.9123$.

\section{III.B Two-Qubit Gates}

In this section we address similar questions as in the previous one, but here we determine the maximal amount of entanglement produced by a general unitary operator acting on two qubits. Note that, contrary to the previous section, we do not allow for local unitary operations during the process. We are also going to determine the initially separable input state which leads to the maximal amount of entanglement of the output state. We will call this state best input state. As measure of entanglement we are going to use (without loss of generality, see Sect. II.B) the concurrence (4).

In [6] we showed that for any unitary operator $U_{A B}$ there exist local unitary operators, $U_{A}, U_{B}, V_{A}, V_{B}$, and a non-local unitary operator $U_{d}$ such that

$$
U_{A B}=U_{A} \otimes U_{B} U_{d} V_{A} \otimes V_{B},
$$

where

$$
U_{d}=e^{-i \sigma_{A}^{\mathrm{T}} d \sigma_{B}}
$$

and $d$ is a diagonal matrix. We will denote the diagonal elements of $d$ by $\alpha_{1}, \alpha_{2}, \alpha_{3}$. Note that any measure of entanglement is not changed under local unitary operators. Thus the entanglement created by $U_{A B}$ is the same as the one created by $U_{d} V_{A} \otimes V_{B}$. And so the maximal amount of entanglement which can be produced by applying a general unitary $U_{A B}$ is the same as the one created by the corresponding operator $U_{d}$. This means that we have to deal with unitary operators which are determined by only 3 parameters, $\alpha_{1}, \alpha_{2}, \alpha_{3}$, instead of 15 parameters, which are required in order to describe a general (special) unitary operator acting on two qubits.

When studying the maximum amount of entanglement created by a two-qubit gate we can restrict ourselves to the case where

$$
\pi / 4 \geq \alpha_{1} \geq \alpha_{2} \geq \alpha_{3} \geq 0 .
$$

This is due to the fact that the maximal amount of entanglement created by $U_{d}$ is symmetric around $\pi / 4$ and $\pi / 2$-periodic in $\alpha_{1}, \alpha_{2}$, and $\alpha_{3}$ [6].

It can be easily shown that the operator $U_{d}$ is diagonal in the magic basis, and therefore we can write

$$
U_{d}=\sum_{k=1}^{4} e^{-i \lambda_{k}}\left|\Phi_{k}\right\rangle\left\langle\Phi_{k}\right| .
$$

The phases $\lambda_{k}$ are

$$
\begin{aligned}
& \lambda_{1}=\alpha_{1}-\alpha_{2}+\alpha_{3}, \lambda_{2}=-\alpha_{1}+\alpha_{2}+\alpha_{3}, \\
& \lambda_{3}=-\alpha_{1}-\alpha_{2}-\alpha_{3}, \lambda_{4}=\alpha_{1}+\alpha_{2}-\alpha_{3} .
\end{aligned}
$$

According to the above discussion, we just have to find which states $|\phi\rangle_{A},|\psi\rangle_{B}$ maximize the concurrence of the output state $U_{d}|\phi\rangle_{A}|\psi\rangle_{B}$.

Writing the input and output state in the magic basis with the coefficients $w_{k}, \mu_{k}$ respectively, we apply the unitary operator $U_{d}$ and obtain

$$
\sum_{k} \mu_{k}\left|\Phi_{k}\right\rangle=U_{d}\left(|\phi\rangle_{A}|\psi\rangle_{B}\right)=\sum_{k} w_{k} e^{-i \lambda_{k}}\left|\Phi_{k}\right\rangle
$$

We want to maximize the concurrence of the output state, $C=\left|\sum_{k} \mu_{k}^{2}\right|$, under the following conditions:

(c1) $\sum_{k}\left|\mu_{k}\right|^{2}=1$; that is the output state (and therefore the input state) must be normalized.

(c2) $\sum_{k} \mu_{k}^{2} e^{2 i \lambda_{k}}=0$. This condition is due to the fact that the input state is a product state, which can be seen as follows. From (28) we see that $w_{k}=\mu_{k} e^{i \lambda_{k}}$, and as mentioned in Sect. II.B the input state $\left(\sum_{k} w_{k}\left|\Phi_{k}\right\rangle\right)$ is a product state if the sum of the coefficients in the magic basis squared vanishes.

We can determine the maximum of the concurrence of the output state under the conditions (c1), (c2) by maximizing $C^{2}$ and imposing the above conditions in terms of Lagrange multipliers, i.e. we maximize

$$
\begin{gathered}
f\left(\mu_{1}, \ldots, \mu_{4}\right)=\sum_{k, l} \mu_{k}^{2}\left(\mu_{l}^{*}\right)^{2}-2 \eta_{1}\left(\sum_{k}\left|\mu_{k}\right|^{2}-1\right) \\
-\eta_{2} \sum_{k} \mu_{k}^{2} e^{2 i \lambda_{k}}-\eta_{2}^{*} \sum_{k}\left(\mu_{k}^{*}\right)^{2} e^{-2 i \lambda_{k}}
\end{gathered}
$$

where $\eta_{1}$ is real. The result of this maximization is the following [6]:

(a) If $\alpha_{1}+\alpha_{2} \geq \pi / 4$ and, at the same time, $\alpha_{2}+\alpha_{3} \leq$ $\pi / 4, U_{d}$ is able to create a maximally entangled state. 
The input states which lead to a maximally entangled output state follow from this maximization [6].

(b) Otherwise we have that the largest reachable concurrence is

$$
C=\max _{k, l}\left|\sin \left(\lambda_{k}-\lambda_{l}\right)\right| .
$$

Except for global phases the corresponding output state is $1 / \sqrt{2}\left(\left|\Phi_{k}\right\rangle+i\left|\Phi_{l}\right\rangle e^{\lambda_{k}-\lambda_{l}}\right)$, and the separable input state, which leads to this maximum is

$$
\frac{1}{\sqrt{2}}\left(\left|\Phi_{k}\right\rangle+i\left|\Phi_{l}\right\rangle\right)
$$

Note that in the case $\alpha_{1} \leq \pi / 8$, we obtain that $C=\sin \left(\alpha_{1}+\alpha_{2}\right)$, which is directly related to the entanglement capability of the Hamiltonian of the form $\sigma_{A}^{\mathrm{T}} d \sigma_{B}$, ( $h_{\max }$ would be $\alpha_{1}+\alpha_{2}$ in this case) in the previous subsection. For higher values of $\alpha_{1}$ the result may not be directly related to that quantity.

\section{Using Ancillas}

Here we analyze the two scenarios described in the previous section, where we now allow Alice and Bob to use auxiliary systems. So, we consider the situation where Alice and Bob have two qubits each*. We will call a state locally entangled if the two qubits at $\mathrm{Al}$ ice's or Bob's side are entangled. If none of the qubits is entangled we will call the corresponding state a local product state. Analogously to the previous section we apply a general non-local Hamiltonian (two-qubit gate) on one of Alice's qubits and one of Bob's. As shown in Sect. III we can, while maximizing the entanglement rate, restrict ourselves to Hamiltonians of the form (20) and unitary operators of the form (24) with the restrictions (25). The main difference between this scenario and the one described in the previous section is that the states no longer belong to a Hilbert space of two qubits. Here we have to deal with higher dimensional Hilbert spaces and therefore, as explained in Sect. II.B, the measures of entanglement are not monotonic functions of each other. We will show that this implies that the question, which input state is the best input state, is not well-defined. Thus, in order to analyze this problem we first of all

"Note that, since one of Alice' and Bob's subsystems (the ones on which they apply the unitary operator) are qubits, the state describing such a subsystem and an ancilla of arbitrary dimension can always be viewed as a state describing two qubits (it has at most two Schmidt coefficients). have to specify according to which measure of entanglement we want to determine the best input state. Once we have specified the measure of entanglement it is possible to answer the same questions as in the previous section.

Let us start now by comparing some measures of entanglement. We consider some special unitary operators, fix the measure of entanglement, and determine the best input state. Summarizing the results obtained by numerical procedures, we have that:

- Schmidt number: Locally entangled states are always "better" than local product states. This can be easily understood since in the first case the maximum value which $E_{\mathrm{S}}$ can take is 3 , whereas in the latter one it can be at most 1 . Thus, using this measure of entanglement the ancillas will, in general, increase the entanglement of the output state.

- Rènyi entanglement: We have checked that for this measure the best input states are always either local product states or local maximally entangled states. In particular, in [6] we provide analytical results for some special cases.

- Entanglement monotones: We have verified that there are unitary operators $U_{d}$ for which local product states are the best input states, whereas for some others the local maximally entangled states lead to the most entangled output state. However, there also exist some $U_{d}$ for which neither the product state nor the maximally entangled state is the best input state.

From these examples it becomes clear that it does not make much sense to ask for the best input state, if one does not specify according to which measure of entanglement.

So, let us specify now the measure of entanglement according to which we want to maximize the entanglement production. We calculate the maximum entanglement rate using the entropy of entanglement. Then, it is possible to show that [5] if $\gamma_{3} \neq 0$, the use of ancillas can help to increase the maximum rate of entanglement $\Gamma_{\max }$ as well as the rate $\Gamma_{E}$ for a given entanglement $E$ of state $|\Psi\rangle$.

It is also possible to calculate the best input state for a given measure of entanglement for certain twoqubit gates. For instance in [6] we showed that for $U_{d}$ with $\alpha=\alpha_{1}=\alpha_{2}=\alpha_{3}$ the input states which lead to the most entangled output state according to the Rènyi entanglement are, depending on $\alpha$, either locally maximally entangled states or local product states: for small values of $\alpha$ the ancillas do not help at 
all, whereas for larger $\alpha$ 's the use of them increases the Rènyi entanglement of the output state.

\section{Non-unitary Processes}

So far, we have shown how much entanglement can be created by a general unitary operator, with or without using local unitary operators during the process. It is natural to ask now which physical interactions, in general, are able to create entanglement. In [7] we answered this question.

Mathematically, a physical action can always be described as a completely positive linear map (CPM), which we will denote by $\mathcal{E}$. That is a map which transforms a positive operator into a positive operator, no matter which extension is used [15]. We will show now that there exists a one-to-one correspondence between completely positive maps and positive operators (unnormalized density operators) acting on two systems.

Let us consider two systems, $A$ and $B$, spatially separated, each of them composed of two particles $\left(A_{1,2}\right.$, and $\left.B_{1,2}\right)$. We also consider a CPM $\mathcal{E}$ acting on systems $A_{1}$ and $B_{1}$. We are interested in whether this CPM can create "non-local" entanglement between the systems $A$ and $B$ [16]. We define the operator $E_{A_{1} A_{2}, B_{1} B_{2}}$ acting on $\mathcal{H}_{A} \otimes \mathcal{H}_{B}$ [where $\mathcal{H}_{A}=\mathcal{H}_{A_{1}} \otimes \mathcal{H}_{A_{2}}$ and $\mathcal{H}_{B}=\mathcal{H}_{B_{1}} \otimes \mathcal{H}_{B_{2}}$, and $\left.\operatorname{dim}\left(\mathcal{H}_{A_{i}}\right)=\operatorname{dim}\left(\mathcal{H}_{B_{i}}\right)=d\right]$ as follows:

$$
E_{A_{1} A_{2}, B_{1} B_{2}}=\mathcal{E}\left(P_{A_{1} A_{2}} \otimes P_{B_{1} B_{2}}\right) .
$$

Here, $P_{A_{1} A_{2}}=|\Phi\rangle_{A_{1} A_{2}}\langle\Phi|$, where $|\Phi\rangle_{A_{1} A_{2}}$ is defined in (1). In the definition (32) the map $\mathcal{E}$ is understood to act as the identity on the operators acting on $\mathcal{H}_{A_{2}}$ and $\mathcal{H}_{B_{2}}$. The operator $E$ has a clear interpretation since it is proportional to the density operator resulting from the operation $\mathcal{E}$ on systems $A_{1}$ and $B_{1}$ when both of them are prepared in a maximally entangled state with two auxiliary systems, respectively.

On the other hand, one can easily show that

$$
\begin{aligned}
\mathcal{E}\left(\rho_{A_{1} B_{1}}\right)= & d^{4} \operatorname{tr}_{A_{2} A_{3} B_{2} B_{3}} \\
& \cdot\left(E_{A_{1} A_{2}, B_{1} B_{2}} \rho_{A_{3} B_{3}} P_{A_{2} A_{3}} P_{B_{2} B_{3}}\right) .
\end{aligned}
$$

This equation also has a very simple interpretation. It reflects the fact that if we have the state $E_{A_{1} A_{2}, B_{1} B_{2}}$ at our disposal, we can always produce the map $\mathcal{E}$ on any state of systems $A_{3}$ and $B_{3}$ by performing a joint measurement locally such that both systems
$A_{2} A_{3}$ and $B_{2} B_{3}$ are projected onto the maximally entangled state (1). Of course, this will happen with certain probability.

The relations (32) and (33) induce a linear one-toone correspondence between CPM acting on tensor product spaces and positive operators. In fact, this correspondence can be viewed as an extension of the isomorphism introduced by Jamiolkowski [15] to tensor product spaces. Using these relations it is very easy to show that:

(i) $\mathcal{E}$ is separable if $E_{A_{1} A_{2}, B_{1} B_{2}}$ is separable with respect to the systems $\left(A_{1} A_{2}\right)$ and $\left(B_{1} B_{2}\right)$. Thus, we can study the separability of CPM by studying the problem of separability of positive operators. This immediately implies that we can use all the results derived for the latter problem [17].

(ii) $\mathcal{E}$ can create non-local entanglement between $A$ and $B$ if $E_{A_{1} A_{2}, B_{1} B_{2}}$ is non-separable with respect to the systems $\left(A_{1} A_{2}\right)$ and $\left(B_{1} B_{2}\right)$. In particular, we can always obtain a state whose density operator is proportional to $E_{A_{1} A_{2}, B_{1} B_{1}}$ out of separable states by entangling our systems locally with ancillas.

(iii) If $\mathcal{E}$ corresponds to a unitary action, the corresponding operator has rank one, i. e. it can be written as $E=|\Psi\rangle\langle\Psi|$, where $|\Psi\rangle \in \mathcal{H}_{A_{1}} \otimes \mathcal{H}_{A_{2}} \otimes \mathcal{H}_{B_{1}} \otimes \mathcal{H}_{B_{2}}$ is a normalized state.

Using this isomorphism it is possible to calculate the amount of entanglement which is used in order to implement a non-local operation. There are many other important applications of this powerful isomorphism. For instance, as shown in [18] it is possible to prove that non-local unitary operations can be purified, stored, or compressed.

\section{Conclusion}

In this paper we have shown how one can entangle two systems in an optimal way using two qubit interactions. On the one hand, we have studied the maximum entanglement rate obtainable with a nonlocal Hamiltonian. We have allowed for local unitary operations during the process, in order to increase the entanglement production. On the other hand, we have studied the situation where we use a two-qubit gate to entangle the systems as much as possible. Here we have not allowed for intermediate operations. In both cases we have determined the input state which leads to this maximal production of entanglement. Further- 
more we have analyzed the situation where both parties use auxiliary systems. We have shown that in this case the question for the best input state is not welldefined, since the answer depends on the measure of entanglement. We have shown that the entanglement production can be increased with the help of auxiliary systems for particular non-local interactions. We have also determined the physical processes which are able to create entanglement, using an isomorphism between density operators and physical actions. We

[1] A. Einstein, B. Podolsky, and N. Rosen, Phys. Rev. 47, 777 (1935).

[2] E. Schrödinger, Proc. Cambridge Philos. Soc. 31, 555 (1935).

[3] See, for example, the special issue of Fortschritte der Physik "Experimental proposals for quantum computation", 48, Eds. S. Braunstein and H.-K. Lo, and references therein; See also C. A. Sackett, D. Kielpinski, B. E. King, C. Langer, V. Meyer, C. J. Myatt, M. Rowe, Q. A. Turchette, W. M. Itano, D. J. Wineland, and C. Monroe, Nature London 404, 256 (2000), A. Rauschenbeutel, G. Nogues, S. Osnaghi, P. Bertet, M. Brune, J.-M. Raimond, and S. Haroche, Science 288, 2024 (2000).

[4] P. Zanardi, C. Zalka, and L. Faoro, quant-ph/0005031.

[5] W. Dür, G. Vidal, J. I. Cirac, N. Linden, and S. Popescu, quant-ph/ 0006034.

[6] B. Kraus and J. I. Cirac, quant-ph/0011050.

[7] J. I. Cirac, W. Dür, B. Kraus, and M. Lewenstein, Phys. Rev. Lett. 86, 544 (2001).

[8] S. Hill and W. K. Wootters, Phys. Rev. Lett. 78, 5022 (1997). have shown that this isomorphism allows to translate all the properties known for density operators to the one of physical processes.

\section{Acknowledgement}

This work was supported by the Austrian SF, the DFG, the European Community under the TMR network ERB-FMRX-CT96-0087 and project EQUIP (contract IST-1999-11053), the ESF, and the Institute for Quantum Information GmbH.

[9] W. K. Wootters, Phys. Rev. Lett. 80, 2245 (1998).

[10] A. Peres, Quantum Theory: Concepts and Methods, Kluwer Academic Publishers, Dordrecht 1993.

[11] C. H. Bennett, H. J. Bernstein, S. Popescu, and B. Schumacher, Phys. Rev. A 53, 2046 (1996).

[12] G. Vidal, Phys. Rev. Lett. 83, 1046 (1999); M. A. Nielsen, Phys. Rev. Lett. 83, 436 (1999).

[13] R. Horodecki and M. Horodecki, Phys. Rev. A. 54 1838 (1996), and references therein.

[14] G. Vidal, J. Mod. Opt. 47, 355 (2000).

[15] A. Jamiołkowski, Rep. of Math. Phy. No. 4, 3 (1972).

[16] Note that we can always take instead of two particles $A_{1,2}$ a single one with more levels. This is why we are interested in what we call non-local entanglement between systems $A$ and $B$, rather than intrinsic or local entanglement between $A_{1,2}$.

[17] M. Lewenstein, D. Bruss, J. I. Cirac, B. Kraus, M. Kus, J. Samsonowicz, A. Sanpera, and R. Tarrach, quant$\mathrm{ph} / 0006064$ and references therein.

[18] W. Dür and J. I. Cirac, quant-ph/0012148. 